\title{
COMPARATIVE STUDY OF SINGLE AND MULTISLICE COMPUTED TOMOGRAPHY FOR ASSESSMENT OF THE MANDIBULAR CANAL
}

\author{
Adriana da Silva Ferreira PAES ${ }^{1}$, Carla Ruffeil MOREIRA ${ }^{2}$, \\ Marcelo Augusto Oliveira SALES ${ }^{3}$, Marcelo Gusmão Paraíso CAVALCANTI ${ }^{4}$
}

1- Undergraduate student, Department of Radiology, School of Dentistry, University of São Paulo, São Paulo, SP, Brazil.

2- DDS, MSc, PhD Student, Department of Stomatology, Bauru School of Dentistry, University of São Paulo, Bauru, SP, Brazil.

3- Post-Doctoral Fellowship, Department of Radiology, School of Dentistry, University of São Paulo, São Paulo, SP, Brazil; Lauro Wanderley Hospital, Federal University of Paraiba, João Pessoa, PB, Brazil.

4- Professor, Department of Radiology, School of Dentistry, University of São Paulo, São Paulo, SP, Brazil;. Adjunct Assistant Professor, Department of Radiology, College of Medicine, University of Iowa, Iowa City, IA, USA.

Corresponding address: Prof. Dr. Marcelo Cavalcanti - Departamento de Radiologia, Faculdade de Odontologia, Universidade de São Paulo. Avenida Prof. Lineu Prestes 2227, 05508-900 São Paulo, SP, Brasil. Phone: 5511 3091-7807 - Fax: 55 11-3091-7899 - e-mail: mgpcaval@usp.br

Received: December 04, 2006 - Modification: February 28, 2007 - Accepted: April 27, 2007

\begin{abstract}
bjective: The purpose of this study was to evaluate the accuracy of relative measurements from the roof of the mandibular canal to the alveolar crest in multislice (multidetector) computed tomography (MDCT) and single-slice computed tomography (SSCT). Material and Methods: The sample consisted of 26 printed CT films (7 SSCT and 19 MDCT) from the files of the LABI3D (3D Imaging Laboratory) of the School of Dentistry of the University of São Paulo (FOUSP), which had been acquired using different protocols. Two observers analyzed in a randomized and independent order a series of 22 oblique CT reconstructions of each patient. Each observer analyzed the CT scans twice. The length of the mandibular canal and the distance between the mandibular canal roof and the crest of the alveolar ridge were obtained. Dahlberg test was used for statistical analysis.

Results: The mean error found for the mandibular canal length measurements obtained from SSCT was $0.53 \mathrm{~mm}$ in the interobserver analysis, and $0.38 \mathrm{~mm}$ for both observers. On MDCT images, the mean error was $0.0 \mathrm{~mm}$ in the interobserver analysis, and 0.0 and $0.23 \mathrm{~mm}$ in the intraobserver analysis. Regarding the distance between the mandibular canal roof and the alveolar bone crest, the SSCT images showed a mean error of $1.16 \mathrm{~mm}$ in the interobserver analysis and 0.66 and $0.59 \mathrm{~mm}$ in the intraobserver analysis. In the MDCT images, the mean error was $0.72 \mathrm{~mm}$ in the interobserver analysis and 0.50 and $0.54 \mathrm{~mm}$ in the intraobserver analysis. Conclusion: Multislice CT was demonstrated a more accurate method and demonstrated high reproducibility in the analysis of important anatomical landmarks for planning of mandibular dental implants, namely the mandibular canal pathway and alveolar crest height.
\end{abstract}

Uniterms: X-ray computed tomography; Dental Implants; Mandibular nerve.

\section{INTRODUCTION}

The mandibular canal is one of the most important anatomical landmarks in the mandible. Given that the inferior alveolar nerve, artery and vein run inside the mandibular canal, this structure is very relevant to implant procedures due to high risks of neurovascular damage ${ }^{2}$. The mandibular canal presents a course that begins in the mandibular foramen and ends where the mental foramen opens ${ }^{3}$. The mandible has been widely used as an osseointegrated implant receptor site, which makes the anatomical knowledge of this region extremely significant and indispensable to the accurate location of the mandibular canal ${ }^{11}$.

Mandibular implants are now an important field to prosthetic, surgical and orthodontic procedures ${ }^{3}$, and the success or failure of placing dental implants in the mandible is directly linked to the anatomical knowledge of this region as well as to the proper evaluation of bone quality and quantity.

Computed tomography (CT) is considered one of the most valuable imaging modalities for dental implant placement because it allows the acquisition of fast, reproducible and reliable images. It has been shown that CT offers better visualization for dental implant planning than any other radiographic method ${ }^{1,5,6,10,11}$. Image quality was improved with the advent of multislice CT scanning, which allows acquiring more slices in a shorter time, due to multiple detector rows, faster table speeds and the opportunity of 
greatly increasing the speed of data acquisition ${ }^{6}$. More anatomic sites are scanned with thinner slices than those provided by spiral CT commonly used for dental implant planning, ${ }^{6}$, which results in more accurate measurements for placement of fixtures. Previous studies have demonstrated the applicability of single-slice CT for dental implant planning in the mandible and maxilla ${ }^{9,13}$. More recently, measurement accuracy using multislice CT have also been reported $^{4,12}$.

The purpose of this study was to evaluate the accuracy of relative measurements from the roof of the mandibular canal to the alveolar crest in multislice (multidetector) computed tomography (MDCT) and single-slice computed tomography (SSCT).

\section{MATERIALAND METHODS}

The sample consisted of 26 printed CT films (7 SSCT and 19 MDCT) from the files of the LABI-3D (3D Imaging Laboratory) of the School of Dentistry of the University of São Paulo (FOUSP), which had been acquired using different protocols. The protocol to obtain the SSCT images (High speed, General Electric, Milwaukee, WI, USA) consisted of original 2.0-mm-thick single axial slices with a reconstruction interval of $1.0 \mathrm{~mm}$ in 1.0 second. The protocol to obtain MDCT images (Aquilion 16, Toshiba Medical, Tustin, CA, USA) consisted of original 0.5-mm-thick axial slices with a reconstruction interval of $0.3 \mathrm{~mm}$ in 0.5 second. Both matrix resolutions were 512 X 512 pixels. The presence of local pathologies was an exclusion criterion for sample composition.
Subsequently, oblique cuts were obtained from original axial images, according to the following protocols: $1.0 \mathrm{~mm}$ slice thickness by $2.0 \mathrm{~mm}$ between the oblique cuts for SSCT, and $1.0 \mathrm{~mm}$ thickness by $1.0 \mathrm{~mm}$ between the oblique cuts for MDCT.

All images were analyzed on a light-box using a 3X magnifying glass. Two previously calibrated observers analyzed twice each CT image in a randomized and independent order with 2 -week interval between the analyses.

The measurements referring to mandibular canal extension were determined by the number of oblique cuts from the mandibular foramen to the mental foramen opening multiplied by the interval between the oblique cuts. For the SSCT protocol, the measurements were multiplied by 2 in order to determine the mandibular canal extension. The same landmarks were used for the MDCT protocol (Figure 1).

The distance between the mandibular canal roof and crest of the alveolar ridge was determined by a series of 22 oblique cuts from each patient. After demarcation of the mentioned structures with a permanent marker pen (Pilot ${ }^{\circledR}$ do Brasil, fine point $1.0 \mathrm{~mm}$ ) on an acetate paper sheet set over the film, the data were transferred to the scale present in the CT film in order to achieve the real measurement. The criterion used for selection of the sites to be measured was the existence of edentulous regions in an interval of 10 consecutive cuts on both right and left mandibular sides.

The intra and interobserver data were analyzed statistically using the Dahlberg error test.
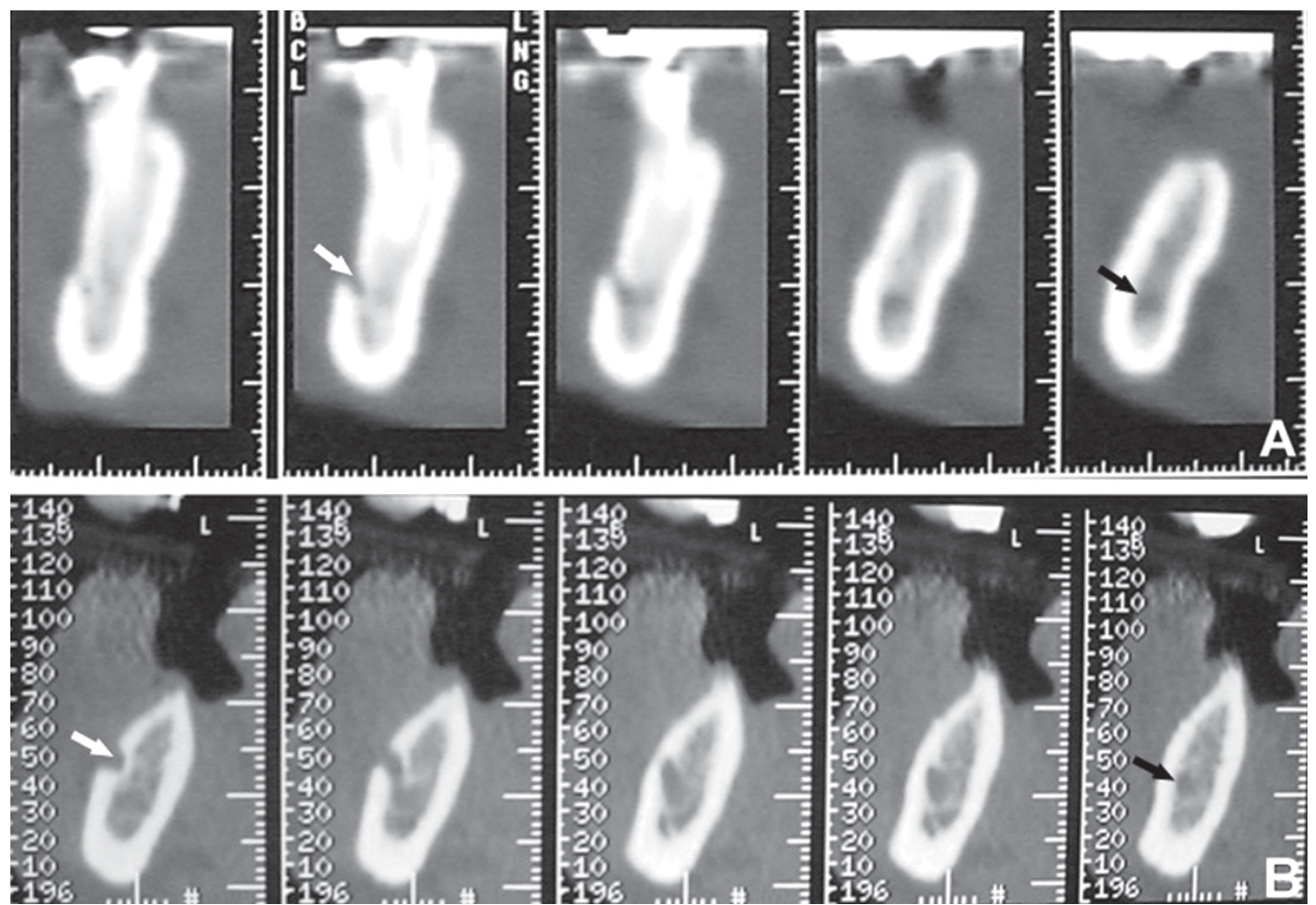

FIGURE 1- A. Single-slice CT cross-section of the mandible. B. Multislice CT showing better depiction of the anatomical landmarks (white arrows - mental foramen opening; black arrows - mandibular canal) 


\section{RESULTS}

A total of 154 measurements from SSCT and 418 from MDCT images were obtained. Part of the measurements obtained by the observers is plotted on Figures 2 and 3 . These figures illustrate the variation between the values obtained from observers 1 and 2, showing the existence of higher values in SSCT images.

Regarding the mandibular canal extension measurements, the mean errors in SSCT images were $0.53 \mathrm{~mm}$ in the interobserver analyses and $0.38 \mathrm{~mm}$ for both observers individually. In MDCT images, these values were $0.0 \mathrm{~mm}$ in the interobserver analyses, and 0.0 and $0.23 \mathrm{~mm}$ in the intraobserver analyses. (Table 1)

When the distance between the mandibular canal roof and the alveolar bone crest was measured, SSCT presented a $1.16 \mathrm{~mm}$ mean error in the interobserver analyses and 0.66 and $0.59 \mathrm{~mm}$ in the intraobserver analyses. In MDCT images, the mean errors were $0.72 \mathrm{~mm}$ in the interobserver analyses, and 0.50 and $0.54 \mathrm{~mm}$ in the intraobserver analyses (Table 1). The mean differences in interobserver measurements were $0.30 \mathrm{~mm}$ and $0.25 \mathrm{~mm}$ for SSCT and MDCT, respectively.

\section{DISCUSSION}

Since the very early development of dental implants, conventional radiographic techniques (periapical, panoramic and occlusal radiographs and teleradiography) have not been deemed completely reliable for assessment of areas for implant placement ${ }^{2}$.

Cross-sectional images are ideal sources of preoperative information because they provide more information regarding the appearance, location and course of the anatomical structures in the jaws ${ }^{9}$. They also provide the mandibular canal location and allow measuring the height, width and angle of the alveolar process. Conventional tomography was initially employed to obtain oblique cuts but it has some limitations, such as a considerable distance between the tomographic slices and image overlapping, which result in very blurry images ${ }^{15}$.

Lindh, et al. ${ }^{10}$ (1995) stated that the tomographic examination surpasses the radiographic assessment because it shows the relation between the mandibular canal and the cortical bone in a buccolingual direction. While investigating the reliability and validity of linear tomography, Butterfield, et al. ${ }^{3}$ (1997) worked with anatomical measurements made by 7 observers on tomographic scans of 5 edentulous jaws. The authors found statistically significant interobserver and intraobserver variations regarding mandibular canal delimitation and the determination of the distance between the canal roof and the alveolar ridge.

Klinge, et al. ${ }^{8}$ (1989) measured the distance from the crest of the alveolar ridge to the mandibular canal roof in macroscopic sections and compared to the distance obtained with periapical radiography, panoramic radiography, hypocycloidal tomography and CT images. They concluded that CT provided the most accurate location of the mandibular canal and is therefore probably the best method for preoperative planning of dental implant surgery involving the area close to the mandibular canal.

There are two modes of CT scan: step-and-shoot CT (called conventional CT) and spiral $\mathrm{CT}^{7}$. During CT development, technologic advances led to a remarkable improvement in spatial resolution and scanning times ${ }^{15}$. The resolution of a CT scan depends on several factors of which the most important are the size of each dot or picture element, CT slice thickness and the distance between slices ${ }^{15}$. Conventional CT has a poor scanning efficiency that directly limits its performance. Spiral CT was introduced in the 1990's to improve CT scanning outcomes. ${ }^{7}$

Preda, et al. ${ }^{13}$ (1997) compared spiral and conventional CT for plannning of dental implant placement and found that spiral CT was more reliable. Spiral CT has been established as a new technology, with faster acquisition of images improving the 3D reconstruction with richer details and better detection of anatomical structures, compared to conventional computed tomography. The 3D-CT images yield highly reliable measurements, which are more suitable for treatment evaluation and planning, and may improve implant placement for providing more precise information about the mandibular canal, incisive foramen and alveolar process location ${ }^{6}$.

Spiral CT scanner may have a single-row detector array (SSCT) or a special system equipped with a multirow detector array (MDCT) ${ }^{7}$. After establishment of SSCT as a reliable method for quantitative and qualitative analyses of anatomic structures for safe dental implant placement ${ }^{9,13}$, improvements in acquisition methods, including the advent of MDCT, represented an advance in the technology for evaluation of the bone condition.

Compared to SSCT, MDCT is intrinsically more complex and introduces several new concepts ${ }^{7}$. MDCT allows highquality image acquisition from thinner slices (0.5-mm-thick) than those obtained for SSCT ${ }^{11}$, which implies greater accuracy of reproduction of anatomical structures ${ }^{14}$. The results of the present study (Table 1) show that MDCT depicted sharper images, allowing better analyses of the mandibular canal morphology, which implies in greater reliability for radiological evaluation and surgical procedures.

Cavalcanti, et al. ${ }^{4}$ (2002) have demonstrated that the 3D MDCT allows highly accurate measurements for dental implant placement. They found a mean difference in interobserver measurements of about $0.38 \mathrm{~mm}$ and considered it as an acceptable level of reproducibility. Our results showed mean differences in interobserver measurements of $0.30 \mathrm{~mm}$ and $0.25 \mathrm{~mm}$ for SSCT and MDCT, respectively. Therefore, both SSCT and MDCT yielded a precise location of the mandibular canal with high level of inter and intra-observer agreement, MDCT being more accurate.

MDCT provides an excellent visualization and delineation of mandibular anatomy, which, in turn, allows establishing the buccolingual position and height of neurovascular bundle, as well as, evaluating the amount of 


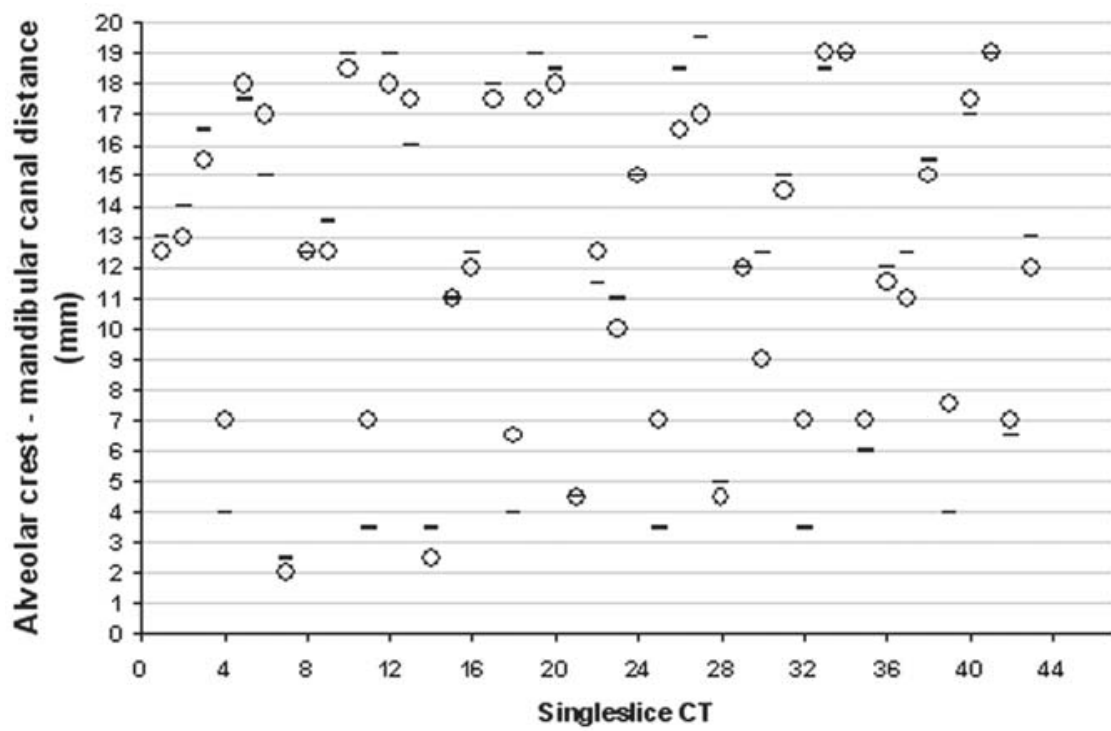

Observer 1
- Observer 2

FIGURE 2- Measurements (in $\mathrm{mm}$ ) from the alveolar crest to the mandibular canal roof obtained by the observers in singleslice CT (partial sample)

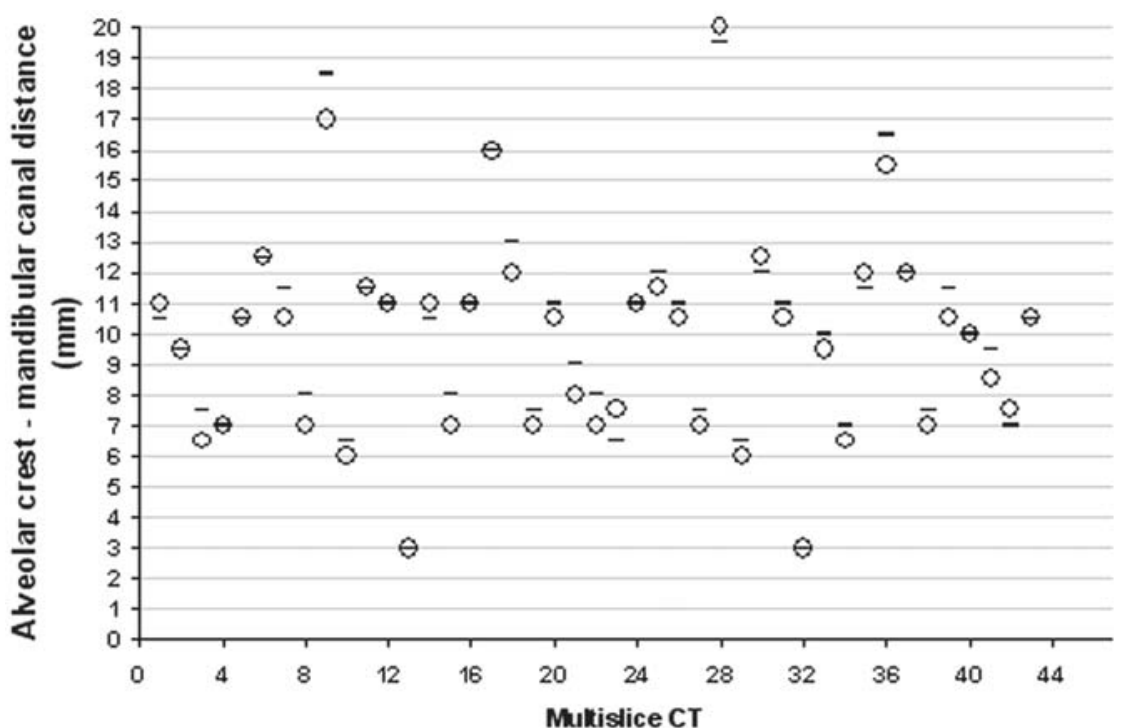

FIGURE 3- Measurements (in $\mathrm{mm}$ ) from alveolar crest to mandibular canal roof obtained by the observers in multislice CT (partial sample)

TABLE 1- Intra and interobserver mean errors (Dahlberg Error) in single-slice and multislice CT

\begin{tabular}{llll} 
Measurements & Analysis & \multicolumn{2}{c}{ Dahlberg error test (mm) } \\
Multislice CT \\
\hline \multirow{2}{*}{ Mandibular canal extension } & & Single-slice CT & 0.0 \\
& Interobserver & 0.53 & 0.0 \\
Alveolar crest-roof canal distance & Intraobserver 1 & 0.38 & 0.23 \\
& Intraobserver 2 & 0.38 & 0.72 \\
& Interobserver & 1.16 & 0.50 \\
\hline
\end{tabular}


bone available for correct placement of implant fixtures. Our data showed that there were more interobserver and intraobserver agreements between the measurements taken when MDCT was used, which means that this image modality provided a clearer and more consistent visualization of inner mandibular structures with excellent depiction of the anatomical landmarks.

Figures 2 and 3 show that measurements made from MDCT images were more accurate, despite intra individual variation. As variation between evaluations is an expected result, these differences (less than $1 \mathrm{~mm}$ in MDCT) are not significant in clinical practice, which assigns to this image modality an important role in surgical planning. MDCT was able to provide high interobserver and intraobserver reproducibility regarding measurements of the alveolar crest height and mandibular canal size and should be preferred to SSCT.

\section{CONCLUSION}

Multislice CT was demonstrated a more accurate method and demonstrated high reproducibility in the analysis of important anatomical landmarks for planning of mandibular dental implants, namely the mandibular canal pathway and alveolar crest height.

\section{ACKNOWLEDGEMENTS}

To CNPq/PIBIC (Undergraduate student scholarship, Paes ASF), CAPES (PhD student scholarship, Moreira CR) and FAPESP (Sales MAO, 2006/05251-8 - Post-Doctoral Fellowship).

\section{REFERENCES}

1- Abrahams JJ. Anatomy of the jaw revisited with a dental CT software program. AJNR Am J Neuroradiol. 1993;14(4):979-90.

2- Berberi A, Mani J, Nasseh I. Duplicated mandibular canal: report of a case. Quintessence Int. 1994;25(4):277-81.

3- Butterfield KJ, Dagenais M, Clokie C. Linear tomography’s clinica accuracy and validity for presurgical dental implant analysis. Oral Surg Oral Med Oral Pathol Oral Radiol Endod. 1997;84(2):203-9.

4- Cavalcanti MG, Ruprecht A, Vannier MW. 3D volume rendering using multislice CT for dental implants. Dentomaxillofac Radiol. 2002;31(4):218-23

5- Cavalcanti MG, Yang J, Ruprecht A, Vannier MW. Validation of spiral computed tomography for dental implants. Dentomaxillofac Radiol. 1998;27(6):329-33.

6- DelBalso AM, Greiner FG, Licata M. Role of diagnostic imaging in evaluation of the dental implant patient. Radiographics. 1994;14(4):699-719.

7- Hu H. Multi-slice helical CT: scan and reconstruction. Med Phys. 1999;26(1):5-18.
8- Klinge B, Petersson A, MALY P. Location of the mandibular canal: comparison of macroscopic findings, conventional radiography, and computed tomography. Int J Oral Maxillofac Implants. 1989;4(4):327-32

9- Liang X, Jacobs R, Lambrichts I. An assessment on spiral CT scan of the superior and inferior genial spinal foramina and canals. Surg Radiol Anat. 2006;28(1):98-104.

10- Lindh C, Petersson A, Klinge B. Measurements of distances related to the mandibular canal in radiographs. Clin Oral Implants Res. 1995;6(2):96-103.

11- McCollough CH, Zink FE. Performance evaluation of a multislice CT system. Med Phys. 1999;26(11):2223-30.

12- Naitoh M, Katsumata A, Nohara E, Ohsaki C, Ariji E. Measurement accuracy of reconstructed 2-D images obtained by multislice helical computed tomography. Clin Oral Implants Res. 2004;15(5):570-4.

13- Preda L, Di Maggio EM, Dore R, La Fianza A, Solcia M, Schifino MR, et al. Use of spiral computed tomography for multiplanar dental reconstruction. Dentomaxillofac Radiol. 1997;26(6):327-31.

14- Prokop M. Multislice CT: technical principles and future trends. Eur Radiol. 2003;13(Suppl 5):M3-13.

15- Schwarz MS, Rothman SL, Chafetz N, Rhodes M. Computed tomography in dental implantation surgery. Dent Clin North Am. 1989;33(4):555-97. 